\title{
Novel Environments Enhance the Induction and Maintenance of Long-Term Potentiation in the Dentate Gyrus
}

\author{
Cyndy D. Davis, Floretta L. Jones, and Brian E. Derrick \\ Department of Biology and Cajal Neuroscience Research Center, The University of Texas at San Antonio, San Antonio, Texas 78249-0662
}

\begin{abstract}
The induction of long-term potentiation (LTP) in the hippocampal formation can be modulated by different behavioral states. However, few studies have addressed modulation of LTP during behavioral states in which the animal is likely acquiring new information. Here, we demonstrate that both the induction and the longevity of LTP in the dentate gyrus are enhanced when LTP is induced during the initial exploration of a novel environment. These effects are independent from locomotor activity, changes in brain temperature, and theta rhythm. Previous exposure to the novel environment attenuated this enhancement, suggesting that the effects of novelty habituate with familiarity. LTP longevity also was enhanced when induced in familiar environments containing novel objects. Together, these data indicate that both LTP induction and maintenance are enhanced when LTP is induced while rats investigate novel stimuli. We suggest that novelty initiates a transition of the hippocampal formation to a mode that is particularly conducive to synaptic plasticity, a process that could allow for new learning while preserving the stability of previously stored information. In addition, LTP induced in novel environments elicited a sustained late LTP. This suggests that a single synaptic population can display distinct profiles of LTP maintenance and that this depends on the animal's behavioral state during its induction. Furthermore, the duration of LTP enhanced by novelty parallels the time period during which the hippocampal formation is thought necessary for memory, consistent with the view that dentate LTP is of a duration sufficient to sustain memory in the hippocampal formation.
\end{abstract}

Key words: long-term potentiation (LTP); dentate gyrus; novelty; theta rhythm; hippocampus; fascia dentata

\section{Introduction}

Information flow through the "trisynaptic circuit" of the hippocampal formation is dynamic and can vary depending on the animal's behavioral state (Winson and Abzug, 1978a,b; Buzsaki et al., 1981). Similarly, studies of the effect of distinct behavioral states on the induction of long-term potentiation (LTP), a phenomenon thought to reflect a cellular mechanism involved in learning (Bliss and Gardner-Medwin, 1973; Bliss and Lomo, 1973; Bliss and Collingridge, 1993), suggest that behavioral states such as alert waking states and rapid eye movement sleep are more conducive to the induction of LTP than other states (JonesLeonard et al., 1987; Bramham and Srebro, 1989). However, in these previous studies, behavioral states were broadly defined, such as "alert" states or awake and sleep states. Other studies observed LTP induction in waking states in which the animal was acquiring new information (Roman et al., 1987; Chaillan et al., 1996). These studies suggest that behaviors involving learning are

Received Nov. 5, 2003; revised May 27, 2004; accepted June 1, 2004.

This work was supported by National Institute on Drug Abuse Grants DA 11983 and GM 60655 (F.L.J.).

Correspondence should be addressed to Dr. Brian E. Derrick, Cajal Neuroscience Research Center, Department of

Biology, University of Texas at San Antonio, 1600 North Loop 1604 West, San Antonio, TX 78249-0662. E-mail: bderrick@utsa.edu.

C.D. Davis's present address: Saneron CCEL Therapeutics, Inc., UTCII3650 Spectrum Boulevard, Suite 170, Tampa, FL 33612.

DOI:10.1523/JNEUROSCI.4970-03.2004

Copyright $\odot 2004$ Society for Neuroscience $\quad$ 0270-6474/04/246497-10\$15.00/0 permissive for LTP induction (Roman et al., 1987; Chaillan et al., 1996). Thus, behavioral states that involve associative learning appear permissive for LTP induction.

One behavior that implicitly involves learning and that may be particularly permissive for hippocampal synaptic plasticity is the exploration of enriched or "novel" environments (Rosenzweig et al., 1962; O'Keefe and Nadel, 1978; Green and Greenough, 1986; Wilson and McNaughton, 1993). Previous studies indicate that a number of important physiological changes occur during the initial period of exploring a novel environment, some of which can facilitate LTP induction. For example, a decrease in somatic GABAergic inhibition is observed in the dentate gyrus during the initial exploration of a novel environment (Moser, 1995, 1996). Because a reduction in GABAergic inhibition can facilitate LTP induction by enhancing postsynaptic depolarization (Wigstrom and Gustaffson, 1983, 1985), the exploration of novel environments alters hippocampal function in a manner that would facilitate LTP induction. Thus, novel environments appear to elicit a "mode" of hippocampal function, during which LTP induction is optimal. As such, this mode may reflect states of hippocampal operation during which LTP and learning normally occur.

If behavioral states elicited during the exploration of novel environments also elicit conditions optimal for LTP, then it would be expected that these same conditions would be particularly conducive to the experimental induction of LTP. Several recent studies indicate that LTP induction in the CA1 region ( $\mathrm{Li}$ 
et al., 2003) and the longevity of early and late LTP (L-LTP) in the dentate gyrus (Straube et al., 2003a) are enhanced by novelty. However, studies have yet to address the effect of novelty on the induction and maintenance of L-LTP that can persist for days to weeks after its induction. In addition, in previous studies, the effects of novelty on LTP were assessed before or after LTP induction (Straube et al., 2003a,b). We were interested in determining whether LTP induced during a single, brief period of exposure to novel environments alters LTP induction and its subsequent maintenance in the absence of the novel stimuli, because their continued presence may have influenced the apparent longevity of LTP. Here, we report that inducing LTP during the initial exploration of a novel environment results in an enhancement of the LTP magnitude and increased the longevity of LTP to 2 weeks, double its usual duration when induced in the animal's home cage. This effect can be mediated not only by novel environments but by novel objects as well. Some of these data have been reported previously (Davis-Hart and Derrick, 1997, 1999, 2000; Davis-Hart et al., 1998).

\section{Materials and Methods}

Subjects. Forty-one adult male Fisher-344 rats (350-400 gm), 6 months of age (Charles River, Wilmington, MA), were housed individually with a $12 \mathrm{hr}$ light/dark cycle and ad libitum access to food and water. All animals were handled in accordance with the standards established in the Guide for the Care and Use of Laboratory Animals published by the Institute of Laboratory Animal Resources of the National Research Council (United States).

Permanent electrode implantation. Permanent electrodes were implanted in the medial aspect of the angular bundle and the dentate gyrus as described previously (Villarreal et al., 2002). Animals were anesthetized with pentobarbital sodium, and under sterile conditions, a Tefloncoated, stainless-steel recording electrode was placed in the hilar region of the dorsal dentate gyrus (anteroposterior, $-3.5 \mathrm{~mm}$; mediolateral, $-2.0 \mathrm{~mm}$; dorsoventral, $-3.0 \mathrm{~mm}$ ) (Paxinos and Watson, 1982). A bipolar stainless-steel stimulating electrode was placed in the dorsomedial aspect of the angular bundle, which corresponds to perforant path fibers generated primarily from the medial entorhinal cortex (anteroposterior, $-8.1 \mathrm{~mm}$; mediolateral, $-4.1 \mathrm{~mm}$; dorsoventral, $-2.3 \mathrm{~mm}$ ) (McNaughton and Barnes, 1977; McNaughton, 1980). Electrodes were attached to gold amphenol pins, mounted in plastic sockets, and affixed to the skull with jeweler's screws and dental acrylic (Fig. 1A).

In some animals, a stainless-steel guide cannula (23 gauge) sealed at one end with tin solder was implanted in the contralateral dentate gyrus to allow insertion of a temperature probe (Physiotemp, Clifton, NJ) and measurement of brain temperature simultaneously with the collection of evoked responses. Animals were allowed to recover for at least 2 weeks after surgery before the collection of field responses.

Collection of field responses and LTP induction in awake animals. After recovery, field EPSPs were evoked using a current intensity that produced responses $50 \%$ of the maximal slope $(200-600 \mu \mathrm{A})$ as determined by input-output curves for each animal. This current intensity was used for all subsequent stimulation, including daily collection of lowfrequency-evoked responses and high-frequency trains used to induce LTP. Our previous studies indicated that stimulation at $50 \%$ of the maximal intensity induced LTP $\sim 40-60 \%$ of the time (Davis-Hart and Derrick, 1997). Therefore, we used this intensity for LTP induction to increase the sensitivity of detecting any alterations in LTP induction. The magnitude of field EPSP slopes was measured in the averaged response over the 1-3 msec period after response onset. Response magnitudes were measured each day by collecting 10 evoked responses $(0.333 \mathrm{~Hz}$ rate) at the same time of day for each animal. Responses were amplified, filtered $(0.3 \mathrm{~Hz}$ to $10 \mathrm{kHz})$, digitized $(10 \mathrm{kHz})$, and stored for off-line averaging and analysis using commercially available software (DataWave Technologies, Thornton, CO). After collection of baseline responses, LTP was induced using three high-frequency "theta burst" trains (five 10 msec, $400 \mathrm{~Hz}$ bursts delivered at $200 \mathrm{msec}$ interburst intervals per train;

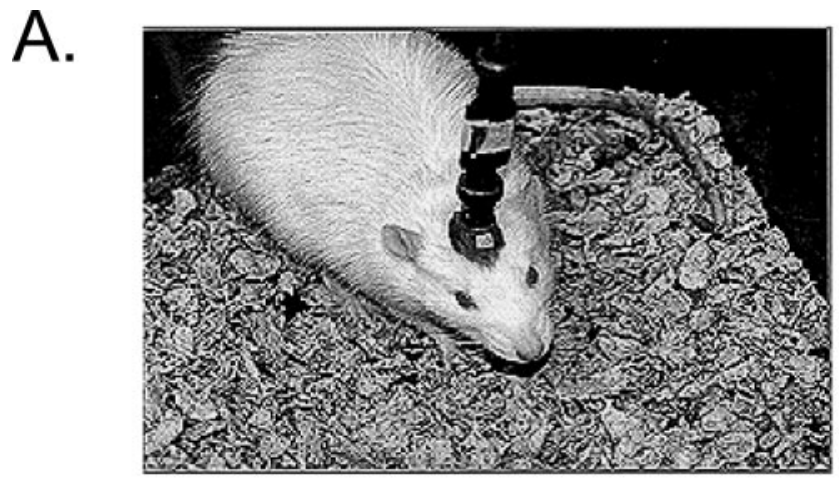

\section{B.}

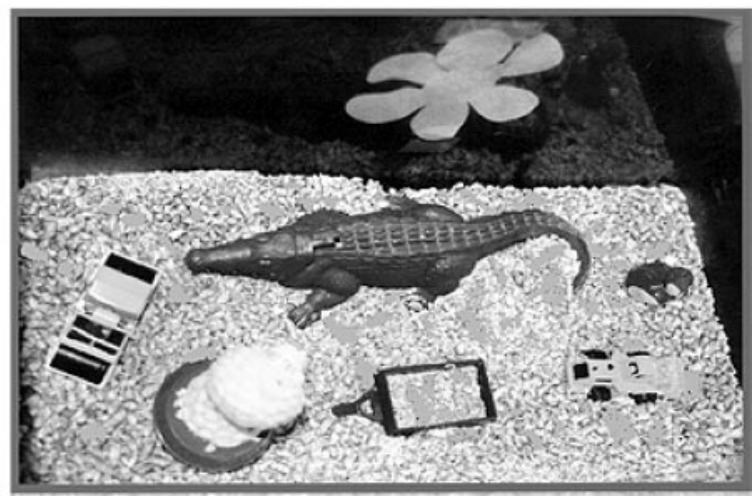

Figure 1. Illustration of preparations used in the present studies. A, Photograph of an adult rat with permanently implanted electrodes. $B$, Photograph of a typical novel environment. Each environment was constructed from a standard cage $(47 \times 25 \times 20 \mathrm{~cm})$ and contained assorted objects (metal and plastic toys) affixed to the cage bottom.

total train duration, $850 \mathrm{msec}$ ) delivered at $5 \mathrm{~min}$ intervals. An EEG was collected ( $1 \mathrm{kHz}$ rate; $2 \mathrm{sec}$ epochs) immediately before and after collection of each low-frequency-evoked response and before and after delivery of each theta burst. The total power of EEG $\left(\mu V^{2}\right)$ in the theta frequency range $(4-12 \mathrm{~Hz})$ was assessed before and after each theta train using a fast Fourier transform analysis (DataWave Technologies). In animals in which hippocampal brain temperature was measured, digitized temperature values were collected simultaneously with each evoked response.

Atropine hydrochloride $(30 \mathrm{mg} / \mathrm{kg}$ ) was used in some experiments and was dissolved in water and administered intraperitoneally during the baseline period and $30 \mathrm{~min}$ before placement in a novel cage. In these experiments, only a single theta burst was used because our previous studies demonstrated that the enhancement of LTP by novelty is equivalent regardless of whether animals receive one or three theta bursts (Davis-Hart and Derrick, 2000; data not shown).

Novel and "familiar" environments. In studies assessing the effects of novel environments on LTP, LTP was induced after the animals were either reoriented in a different location within their home (familiar) cage, or placed in a novel cage. Novel cages were identical to home cages $(47 \times$ $25 \times 20 \mathrm{~cm}$ ), save for the addition of four to five plastic and metal objects, such as open-ended tunnels, miniature toys, and items with non-food odors (e.g., commercial antiseptics). Objects were mounted permanently on the cage floor to prevent displacement by the animal, which can confound measures of total activity, and to maintain specific configurations of objects within the cage (Fig. $1 B$ ).

LTP induction in novel and familiar environments. For all experiments, daily response magnitudes were calculated from an average of 10 responses collected each day in the animal's home cage at a rate of $0.05 \mathrm{~Hz}$. After at least $5 \mathrm{~d}$ of stable daily responses, LTP was induced in either a novel or a familiar environment. For LTP induction, baseline responses were collected in the animal's home cage at a rate of $0.05 \mathrm{~Hz}$ for a mini- 
mum of $15 \mathrm{~min}$. Animals were then gently placed in a novel cage or reoriented in the home (familiar) cage by grasping and moving the animal to the opposite end of the cage so as to control for any effects of handling. We chose to manually place animals in environments, rather than allowing choice entry into the novel environments, because the latter method is often accompanied by avoidance behaviors, and animals frequently do not enter accessible novel cages. In contrast, manually placing animals in cages is likely less stressful because all animals are handled several times a day (handling is necessary to attach head stage connectors necessary for daily data collection). The novel cage bedding was changed, and objects were cleaned with a methanol-based disinfectant after each period of exploration. Responses were again collected over the $15 \mathrm{~min}$ period in the home or novel cage $(0.05 \mathrm{~Hz})$. Locomotor activity was monitored over the $15 \mathrm{~min}$ period after placement or reorientation in a novel or home cage. Five minutes after placement in the novel or home cage, three theta burst trains were delivered at $5 \mathrm{~min}$ intervals. Immediately after delivery of the third and final theta burst delivered $15 \mathrm{~min}$ after placement or reorientation in the cage, animals were grasped and returned to their home cage, or, in the case of home cage controls, grasped and reoriented in their home cage. Responses were again collected in the animal's home cage for an additional $1 \mathrm{hr}$ at the same $(0.05 \mathrm{~Hz})$ rate as baseline responses. Daily responses collected after LTP induction also were collected in the animal's home cage.

Measurement of activity during exploration of novel or familiar environments. Exploratory behaviors include a number of diverse actions, including locomotion, rearing, nose poking, etc. Therefore, we measured the total activity of each animal during recording of daily responses and during the 15 min period of exploration and LTP induction using an activity monitor (Videomex-V; Columbus Institute, Columbus $\mathrm{OH}$ ), which analyzes motion by a pixel-by-pixel analysis of contrast changes within the cage area $\left(1000 \times 3000\right.$ pixels $\left./ \mathrm{cm}^{2}\right)$ over the $15 \mathrm{~min}$ period of exploration. Activity during exploration of novel or familiar environments is expressed as the total number of pixels detecting contrast change over the 15 min period.

Measures of LTP magnitude, probability, and longevity. LTP magnitude was calculated as the percentage change in field EPSP slopes 55-60 min after stimulation compared with the average slope magnitude observed during the $5 \mathrm{~min}$ baseline period immediately preceding placement in home or novel cages. LTP longevity was assessed by averaging 10 responses collected in the rat's home cage $24 \mathrm{hr}$ after LTP induction and daily thereafter for at least 2 weeks. For measures of LTP magnitude over days, the percentage change in the EPSP slope of the averaged daily response was compared with the average magnitude of responses collected during the $3 \mathrm{~d}$ baseline before LTP induction. LTP was considered to have decayed once average daily responses did not differ significantly from the average baseline response magnitude measured over the $3 \mathrm{~d}$ before LTP induction. Although a number of studies quantify LTP decay using exponential curve fitting (Abraham et al., 2002), we have noted that LTP induced in awake animals using a series of theta bursts rarely elicited LTP that appeared to decay exponentially (Villarreal et al., 2002). In fact, on average, LTP magnitude often was larger $24 \mathrm{hr}$ after induction than at $1 \mathrm{hr}$ after tetanus (see Fig. $3 B$ and Results). Therefore, in our studies, LTP longevity was assessed by comparisons with LTP magnitude in control cohorts over the 4-14 d after LTP induction (when LTP decay normally is evident) using a two-way repeated-measures ANOVA and a NewmanKeuls pairwise test for comparisons of LTP magnitude over days. LTP was considered to have "decayed" once the average daily response magnitudes for the group did not differ significantly from the average baseline response amplitude. For all quantitative measures, data presented reflect the mean \pm SEM.

For calculations of LTP induction probability, successful LTP induction was defined arbitrarily as an increase in field EPSP slope amplitudes of $\geq 20 \%$ when measured 55-60 min after tetanus. LTP induction probabilities are presented as the ratio of the number of animals displaying LTP magnitudes of $\geq 20 \%$ over the number of animals displaying changes that were $<20 \%$. However, statistical analyses of probabilities (e.g., Fisher test) require a substantial number of observations for sufficient power. Because our experiments used sample sizes that were rela-
A.

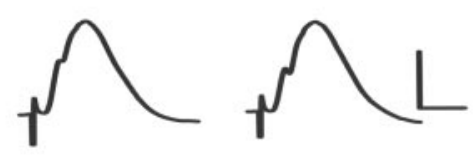

B.

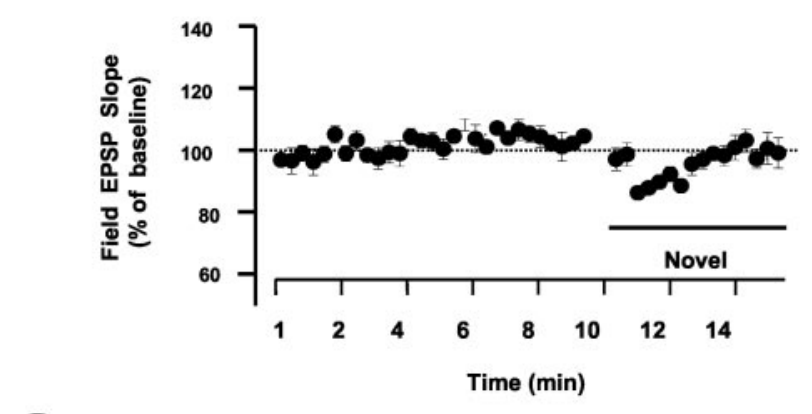

C.

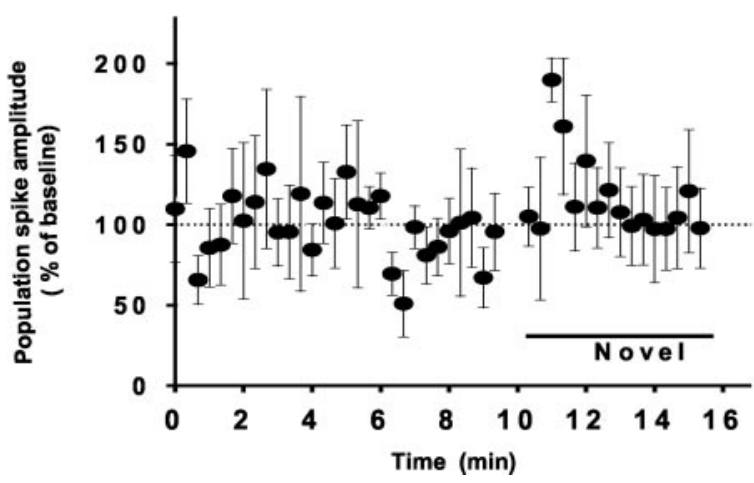

Figure 2. Phasic alterations in evoked perforant path-dentate gyrus responses are observed after displacement of animals to novel or familiar (home) cages. A, Example of perforant path-dentate responses before and after placement in a novel cage showing increases in population spike amplitude and a concomitant decrease in field EPSP slopes. Calibration: 0.5 $\mathrm{mV}, 5 \mathrm{msec}$. Graphs illustrating the average time course and magnitude of changes in field EPSP slopes $(B)$ and population spikes $\pm \operatorname{SEM}(C)$ after placement in a novel environment $(n=5)$ are shown.

tively small ( $<10$ animals/group), LTP induction probabilities are presented here only as a descriptive statistic.

\section{Results}

Effects of novel environments on behavior and perforant path-granule cell synaptic responses

After placement of the animal in the novel environment, the most salient behavioral effect observed was exploratory behavior characterized by ambulation, sniffing, and rearing. Responses that usually accompany fearful or stressful novel environments, such as freezing, defecation, or avoidance behavior (Diamond and Rose, 1994), were not observed. Rather, animals immediately engaged in exploratory behaviors when placed in the cage. Similarly, control animals that were simply grasped and reoriented in a different region of their home cage also showed similar increases in exploratory behaviors (see below).

The one salient effect we observed in perforant path-dentate responses after placement in either a novel or familiar (home) cage was a transient (3-5 $\mathrm{min}$ ) decrease in the field EPSP slope (Fig. $2 A, B)\left(F_{(1,14)}=4.63 ; p<0.06 ; n=5\right)$. This was accompa- 
nied by a paradoxical, intermittent increase in population spike magnitude (Fig. $2 A, C)\left(F_{(1,14)}=4.71 ; p<0.05 ; n=5\right)$. These effects are unlike those observed during extensive exploration and mediated by increases in brain temperature (Erickson et al., 1993; Moser et al., 1993), in which case increases in field EPSPs and decreases in population spikes are observed. However, these effects are quite similar to previous reports indicating altered responsivity of dentate granule cells in response to novel objects (Stanton and Sarvey, 1987; Sara and Segal, 1991; Harley and Sara, 1992; Klukowski and Harley, 1994; Vankov et al., 1995; Kithigina et al., 1997) in which an increase in evoked population spikes are reported. However, a phasic decrease in field EPSPs also was observed when animals were simply reoriented in their home cage (see Figs. $3 A, 5 B$ ). Thus, the effects of exploration and novelty on field EPSPs observed here may reflect a phenomenon distinct from noveltyinduced changes on population spikes, because it was observed during exploration of both novel and familiar environments.

Inducing LTP in novel environments facilitates both induction and longevity

We first examined the effects of inducing LTP during the initial exploration of a novel environment. Five minutes after placement of animals in the novel cage or reorientation in the home cage, three theta burst trains were delivered at 5 min intervals. After $15 \mathrm{~min}$ (immediately after the third train of theta bursts), animals were either returned to their home cage or reoriented again in their home cage. Although the initial level of LTP did not differ between groups immediately after theta bursts (Fig. 3A), LTP magnitude measured 55-60 min after tetanus revealed significantly greater LTP magnitude in animals that received theta bursts in a novel environment $(144 \pm 7.6 \% ; n=7)$ compared with animals receiving theta bursts while exploring their home cage (126 $\pm 1.6 \%$; $F_{(1,13)}=15.44 ; p<0.05 ; n=8$ ) (Fig. $3 A$ ). Using increases in EPSP slopes of $\geq 20 \%$ 55-60 min after tetanus as an arbitrary definition of LTP, potentiation was observed $43 \%$ of the time in the home cage (three of seven stimulation sessions), whereas LTP induced in a novel environment was observed 75\% of the time (six of eight stimulation sessions).

LTP induced during the first encounter with a novel environment not only enhanced LTP magnitude but also increased the longevity of LTP. In these studies, the time course of LTP was assessed by collecting daily responses up to 2 weeks after LTP induction. To ensure an accurate comparison of LTP longevity,
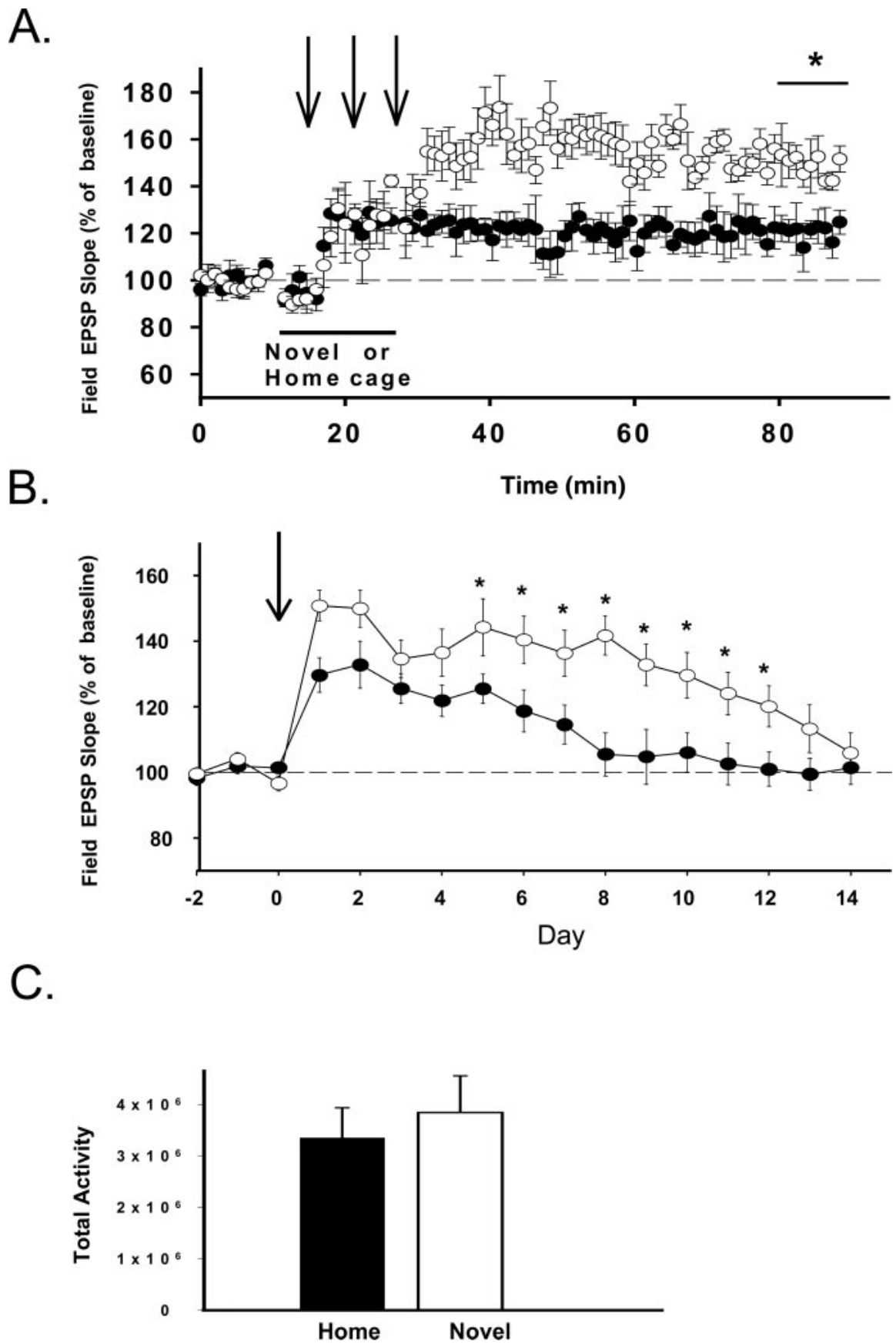

Figure 3. Induction of LTP in novel environments enhances the magnitude and time course of LTP induced with three sets of theta burst trains (arrows). $A$, The magnitude of LTP was enhanced significantly when induced in a novel cage $(O ; n=8)$ compared with LTP induced in the rat's home cage $(\mathbf{O} ; n=7) 1 \mathrm{hr}$ after tetanus $\left({ }^{*} p<0.05\right) . B$, LTP duration over the 2 week period after LTP induction as measured daily in the animal's home cage. Only animals that displayed increases of field EPSP magnitude $>20 \% 1 \mathrm{hr}$ after tetanus were used in comparisons of LTP Iongevity (see Materials and Methods). Comparison of the duration of LTP induced in a novel cage $(O ; n=6)$ or the animal's home cage $(\mathbf{O} ; n=3)$ reveal that LTP longevity was enhanced $5-12 \mathrm{~d}$ after LTP induction in animals tetanized in a novel environment $\left({ }^{*} p<0.05\right)$. C, Measures of activity during exploration of novel or home cages. There were no significant differences in the total activity (total change in pixels within the cage area measured over the 15 min period of exploration) in the home or novel cages ( $p>0.05)$.

only animals showing successful LTP induction $(>20 \%$ increase in field EPSP slopes $1 \mathrm{hr}$ after tetanus) were included in measures of LTP longevity ( $n=6$ for animals tetanized in novel cages; $n=$ 3 for animals tetanized in their home cages). Comparison of the daily magnitude of potentiated responses revealed LTP with a duration of $\sim 7 \mathrm{~d}$ when induced in the home cage, a duration commonly observed for perforant path-dentate LTP (Barnes, 
1979; Villarreal et al., 2002). In contrast, LTP persisted for up to $14 \mathrm{~d}$ when LTP was induced while animals explored a novel environment $\left(F_{(1,7)}=9.8 ; p<0.05\right)$ (Fig. $\left.3 B\right)$. Thus, inducing LTP during periods of exploration of a novel environment facilitates both the induction and longevity of perforant path-dentate LTP.

A number of factors associated with exploratory activity, rather than novel environments per se, also could mediate these alterations in LTP induction and maintenance. One confound associated with recording from awake, freely moving animals is that increased locomotor activity can alter a number of aspects of dentate responses (Moser et al., 1993). Therefore, it is possible that a greater degree of ambulation or exploratory activity in novel environments may have altered LTP induction and its subsequent maintenance. We therefore measured the total activity during the exploration in a subset of animals from each group in these initial experiments. A comparison of the total activity of each animal from each group over the 15 min period of exploration revealed no significant differences in total activity among animals exploring novel and home cages $\left(F_{(1,8)}=0.02 ; p>0.05\right.$; $n=5$ /group) (Fig. 3C).

\section{Novelty-induced facilitation of LTP habituates with} familiarity but is reinstated in a distinct novel environment In our initial experiments, the effects of both novel and familiar (home) environments on LTP were tested in each animal using a counterbalanced experimental design. Over the course of these studies, we noticed that repeated induction of LTP within a single novel environment resulted in a reduction in the enhancing effect of novel environments on LTP (data not shown). Therefore, we addressed directly the effect of familiarity with the novel environment on LTP induction and longevity, as well as the possible contribution of exploratory activity to this effect. In our first experiments, LTP was first induced while the animals explored a novel environment, and the usual facilitation of LTP longevity was observed. Three to 4 weeks later, LTP was induced again while the animal explored this same novel environment. As shown in Figure 4A, LTP longevity was significantly less than the duration of LTP that was induced initially in this same environment $\left(F_{(2,12)}=4.58 ; p<0.05 ; n=5\right)$. Three to 4 weeks later, LTP was induced again in these same animals, with tetani delivered while animals explored a new, unfamiliar novel environment that contained different novel objects. The enhancement of LTP longevity was reinstated by this unfamiliar environment and was identical to LTP longevity observed with the initial novel environment. Measures of activity in separate animals under identical conditions indicate no significant differences in activity while animals explored the novel, familiar novel, or the new novel cages at $3-4$ week intervals $\left(F_{(2,6)}=1.47 ; p>0.05 ; n=4\right)($ Fig. $4 B)$. Together, these data indicate that the enhancement of LTP longevity by novelty habituates with familiarity of the novel environment and that the facilitation of LTP is significantly attenuated when induced while exploring the now familiar novel environment.

\section{Novelty-induced facilitation of LTP induction and LTP longevity is differentially affected by novel environments and novel objects}

The above findings indicate that the facilitation of LTP induced in novel environments is attenuated by familiarity with the environment. One likely explanation for such habituation is that the facilitation of LTP by novel environments may be stimulus bound and dependent on the novel stimuli within the environment. However, there are a number of possible confounds asso-

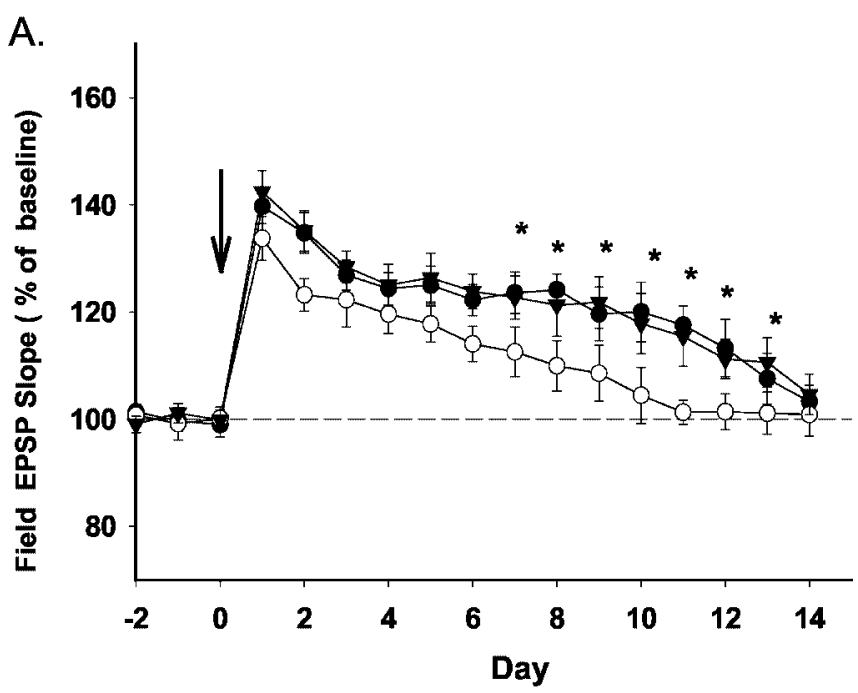

B.

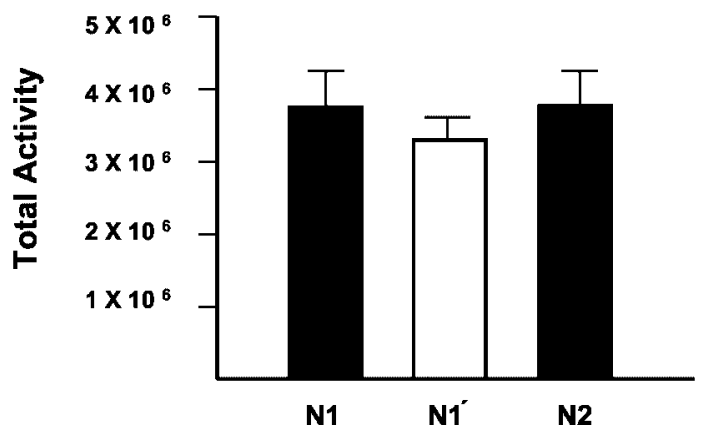

Figure 4. Familiarity with the novel environment attenuates enhanced LTP longevity. $A$, The initial induction of LTP in a novel environment $(\boldsymbol{\nabla} ; n=5)$ produced LTP that persisted for $\sim 14$ $d$. Subsequent induction of LTP in these animals in the same novel environment 3-4 weeks later resulted in a significant reduction in LTP longevity $\left(O ;{ }^{*} p<0.05\right)$, suggesting the animals habituated to the once-novel cage. However, when these same rats were tetanized in a new, unfamiliar novel environment 3-4 weeks later, the novelty-induced enhancement of LTP Iongevity was reinstated (O). B, No significant differences in activity were observed while animals explored novel cages (N1), the same cage 3-4 weeks later (N1'), or a new novel cage 3-4 weeks later (N2; $n=4)$.

ciated with these experiments that also could account for these effects. For example, the effects of novel environments on LTP induction and LTP longevity may be regulated by nonspecific aspects of exploratory behavior, such as ambulation or arousal associated with novelty. It also is possible that contextual or configurational aspects of the objects, rather than simply the novelty of the objects, may be sufficient to elicit conditions that facilitate LTP (Wan et al., 1999; O'Reilly and Rudy, 2001). Thus, some or all of the facilitation effects seen with LTP induced in a novel environment may be mediated by factors other than simply novel stimuli within the environment.

We addressed this question first by testing the effects of novel stimuli on LTP induction and longevity. In these experiments, baseline responses were collected daily for $3 \mathrm{~d}$ in the home cage, after which the animals were placed in a novel cage and allowed to explore this novel environment for 15 min periods over 3 consecutive days $(n=15)$. On the fourth day, the animals were divided into two groups, with one group of animals placed in the original novel cage containing the same, now familiar, objects $(n=9)$ or the standard cage that contained new, unfamiliar 
objects $(n=6)$ (Fig. $5 A)$. LTP was induced using three theta bursts at 5 min intervals after placement in the cages containing the novel or familiar objects. Immediately after LTP induction, animals were returned to their home cages, and responses were collected for 1 additional hour; daily responses were collected in the animal's home cage for 2 weeks after LTP induction.

In contrast to the results of our first study comparing LTP induction in novel environments and the home cage, no significant differences in LTP magnitude as measured at $1 \mathrm{hr}$ were observed among animals tetanized while exploring novel versus familiar objects, although a trend of larger LTP when induced in the presence of the novel objects was observed [change in field EPSP slopes in animals that received theta bursts in the original novel environment, $114 \pm 6 \%(n=9)$; LTP magnitude induced while exploring the novel environment with new objects, $118 \pm$ $8 \%(n=6) ; F_{(1,13)}=0.12 ; p>0.05$ ] (Fig. $\left.5 B\right)$. In addition, the proportion of stimulated animals displaying LTP (increases of $>20 \%$ ) was similar in environments with new and familiar objects (three of six animals for novel objects vs five of nine animals for familiar objects). However, the probability of LTP (increases of $>20 \%$ ) was lower in both groups that were observed in our first experiment in which animals were exposed to a completely novel environment. Thus, novel objects within the cage had little effect on LTP induction, and previous exposure to complex environments reduced the facilitation of LTP magnitude and induction probability normally observed in novel environments. However, an analysis of LTP longevity in a subset of animals displaying LTP ( $n=3$ /group) revealed that the longevity of LTP when induced in environments with novel objects was enhanced compared with animals tetanized in the environment with familiar objects (Fig. $5 C)\left(F_{(1,4)}=8.9 ; p<0.05\right)$. Interestingly, the longevity of LTP induced in the presence of novel objects was similar to the duration of LTP when induced on the initial exposure to a novel environment (compare Fig. $3 B$ ). Again, measures of activity in animals that displayed LTP revealed no differences in total activity on day 4 when animals were tetanized in cages containing familiar or new novel objects $\left(F_{(1,4)}=0.01 ; p>0.05\right.$; $n=6$ ) (Fig. $5 D$ ). Together, these data indicate that the addition of novel objects to a familiar environment significantly enhanced LTP maintenance, but not LTP induction, as reflected in initial LTP magnitude and induction probability.

\section{Does the enhancement of LTP involve alterations in brain temperature or in theta rhythm that occurs with exploratory behaviors?}

A number of factors associated with exploratory behaviors but unrelated to novelty within the environment may have produced the facilitation observed with LTP when induced in novel environments. Previous investigations have reported that extensive ambulation and subsequent increases in brain temperature can alter evoked dentate responses (Moser et al., 1993, 1994). Thus, elevations in brain temperature resulting from greater amounts of ambulation in novel environments could alter induction and subsequent expression of LTP. However, as observed in our original experiments (Figs. 3C, 4B), no differences in total activity were observed among animals exploring either familiar or home cages, or cages containing novel objects. This argues against the view that increased activity underlies the enhancement of LTP when induced in novel environments.

Although the lack of a difference in total activity suggests it is highly unlikely that locomotor-induced changes in brain temperature are a potential confound, we nonetheless determined whether differences in brain temperature might have contributed to the effects observed on LTP induced in novel environments. This was accomplished by measuring the changes in brain temperature during exploration of both the home cage and novel environments using a temperature probe placed in the contralateral dentate gyrus (see Materials and Methods). Both temperature and field EPSP slopes were measured in a separate set of animals $(n=4) 5 \mathrm{~min}$ before and $10 \mathrm{~min}$ after placement in the novel environments or reorientation in the home cage. Brain temperature did not change significantly during exploration of either novel or home cages (Fig. 6).

Another potential factor that we thought would likely alter LTP induction during exploration is the occurrence of theta rhythm, a 3-12 Hz rhythm observed in the hippocampal formation during exploratory behaviors and locomotion (Landfield et al., 1972; Vanderwolf, 1975; Arai and Lynch, 1992). Previous studies indicate that stimulation parameters that mimic theta activity are optimal for inducing long-lived LTP in the CA1 region (Staubli and Lynch, 1987). In addition, LTP induction is enhanced when high-frequency trains are delivered during the positive phase of theta rhythm (Pavlides et al., 1988; Huerta and Lisman, 1993; Orr et al., 2001; Buzsaki, 2002). Thus, it is possible that greater amounts of theta rhythm generated during the periods in novel environments may underlie the enhancement of LTP induction or maintenance. To investigate the possible contribution of theta rhythm to the noveltyinduced enhancement of LTP, we compared EEGs collected before and after each theta burst train in our original experiments in which animals explored either a novel or familiar (home) cage. An analysis of total power in the theta range (3-12 Hz) during two 2 sec epochs before and after each theta burst train revealed no differences in theta power in animals exploring either the home $(n=5)$ or the novel $(n=5)$ environment $\left(F_{(3,19)}=0.002 ; p>0.05\right)$ (Fig. 6B). Thus, activity, brain temperature, and theta rhythm power during tetanus were equivalent among animals exploring novel and familiar environments.

Although the measures of general activity and theta magnitude were equivalent in novel or familiar environments, it remains possible that novel environments may have elicited more frequent, or perhaps longer, periods of theta rhythm over the 15 min period of exploration. We therefore addressed further the possible contribution of theta rhythm to the enhancement of LTP by novelty using systemic administration of atropine sulfate (30 $\mathrm{mg} / \mathrm{kg}$, i.p.), a treatment that blocks type II theta (Vanderwolf and Leung, 1983). In these experiments, only a single theta burst was used to induce LTP. During the course of our studies, we found that the enhancement of LTP magnitude and longevity by novel environment were equivalent regardless of whether one or three theta trains were used (data not shown). In these experiments, atropine $(n=5)$ or water vehicle $(n=5)$ was administered $30 \mathrm{~min}$ before placement in a novel environment, and a single theta train was delivered 5 min later. Although this dose of atropine attenuated baseline responses shortly after administration, atropine did not affect the enhancement of LTP magnitude or longevity when induced in novel environments (Fig. 7A). In addition, both atropine- and vehicle-treated animals showed the usual novelty-induced facilitation of both LTP induction probability (LTP of $>20 \%$ was observed in four of five animals treated with atropine and in four of five animals given only the water vehicle) as well as LTP longevity (compare Figs. 3B, 7B).

\section{Discussion}

The principal finding of these studies is that both the induction and maintenance of LTP are enhanced when LTP is induced while the animal is exploring a novel environment. Novel environments are regarded as rich learning situations and are known to produce a number of alterations in hippocampal morphology, 
A.

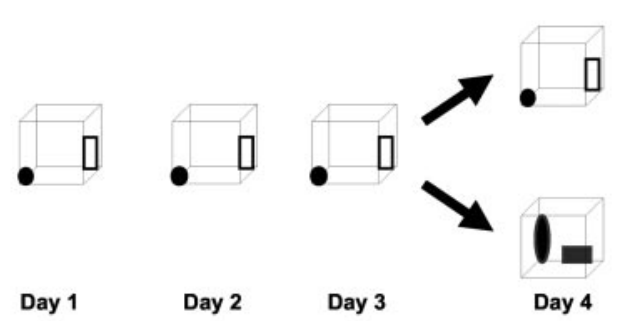

LTP INDUCTION

B.

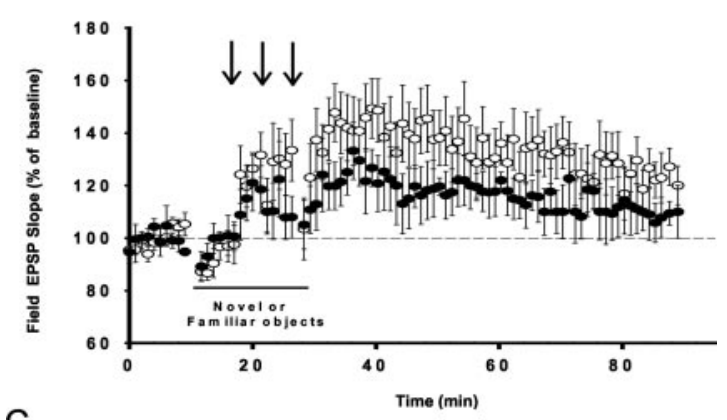

C.

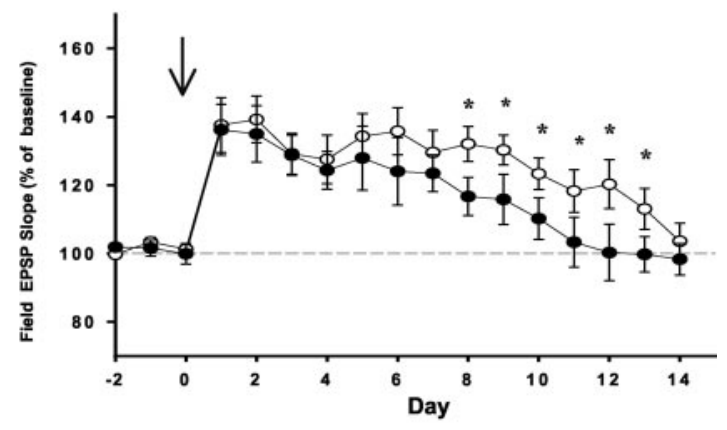

D.

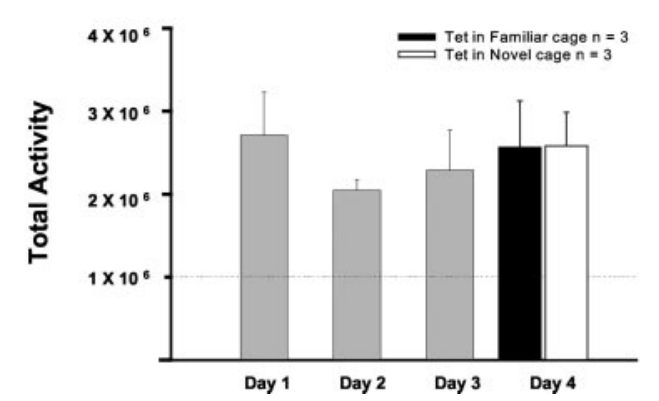

Figure 5. Induction of LTP in the presence of novel objects enhances LTP longevity. A, Graphic showing the experimental design. Animals were allowed to explore a single novel cage for a 15 min period over 3 consecutive days $(n=15)$. On the fourth day, animals were placed either in this same cage or in an identical cage with distinct, unfamiliar objects. LTP was induced by delivering three sets of theta burst trains (arrows). B, Comparison of LTP magnitude at $1 \mathrm{hr}$ when induced in a cage containing familiar $(\mathbf{O} ; n=9)$ or novel $(\bigcirc ; n=6)$ objects. No significant differences were observed in LTP magnitude $1 \mathrm{hr}$ after tetanus $(p>0.05)$. C, The longevity of LTP in animals displaying increases of $>20 \%$ when induced in cages that contained either novel $(n=3)$ or familiar $(n=3)$ objects. LTP longevity was significantly enhanced over days $8-13$ in animals tetanized in the presence of novel objects $\left({ }^{*} p<0.05\right)$. D, Measures of total activity during the 15 min period of exploration over days $1-4$ in animals that displayed LTP. Shown are measures of activity (total change in pixels within the cage area as measured over the 15 min period of exploration; 粼) on days $1-3(n=9)$, and for these same animals placed in home cages with either familiar $(n=3)$ or unfamiliar $(n=6)$ objects on day 4. № significant differences in activity were observed among animals exploring cages with familiar objects ( $\square$ ) or novel objects $(\square)$ on day 4 ( $p>0.05$ ).
A.
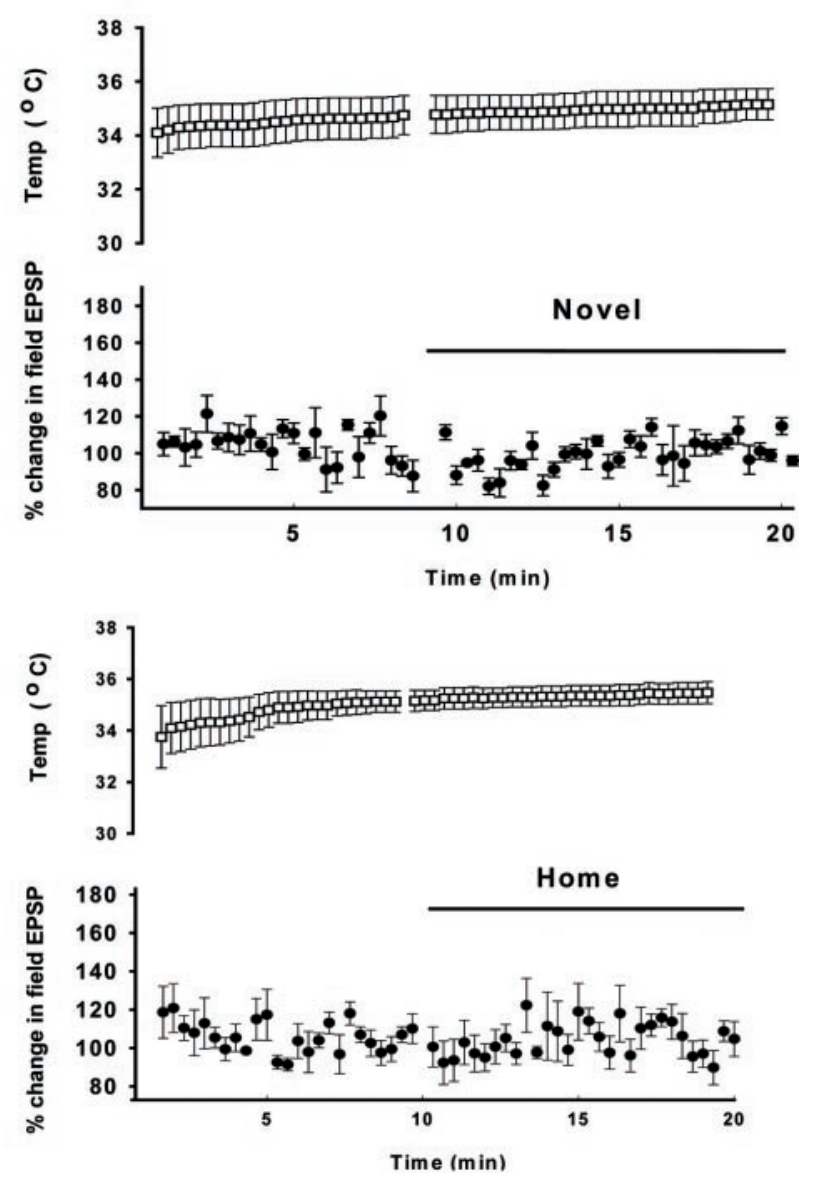

B.

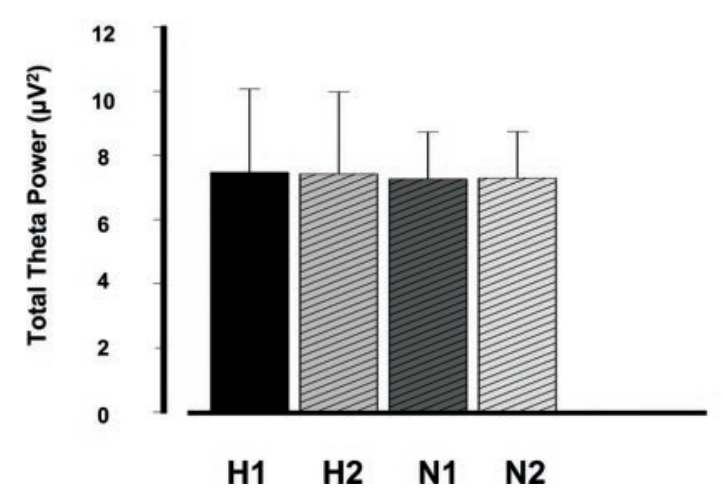

Figure 6. Changes in brain temperature and theta rhythm observed while exploring novel or home environments.A, Plot offield EPSP slopes and brain temperature collected in the home cage and after placement in a novel cage (top) or reorientation within the animal's home cage (bottom). Significant changes in brain temperature were not observed after placement in the novel cage. $B$, Comparison of total power in the theta range $(3-12 \mathrm{~Hz})$ during $\mathrm{LTP}$ induction in animals exploring either novel $(\mathrm{N} ; n=5)$ or home $(\mathrm{H} ; n=5)$ cages. Total theta power was measured in each animal over two 1 sec epochs immediately before (N1, H1) and immediately after (N2, H2) delivery of each theta burst train. No significant differences in total theta power were observed during delivery of theta bursts among animals exploring either the home cage or novel cages $(p>0.05)$.

physiology, and function (Rosenzweig et al., 1962; Sharp et al., 1985; Wilson and McNaughton, 1993). The present study indicates that such conditions not only facilitate LTP induction but also elicit LTP that is particularly long lasting. If LTP is indeed 
A.

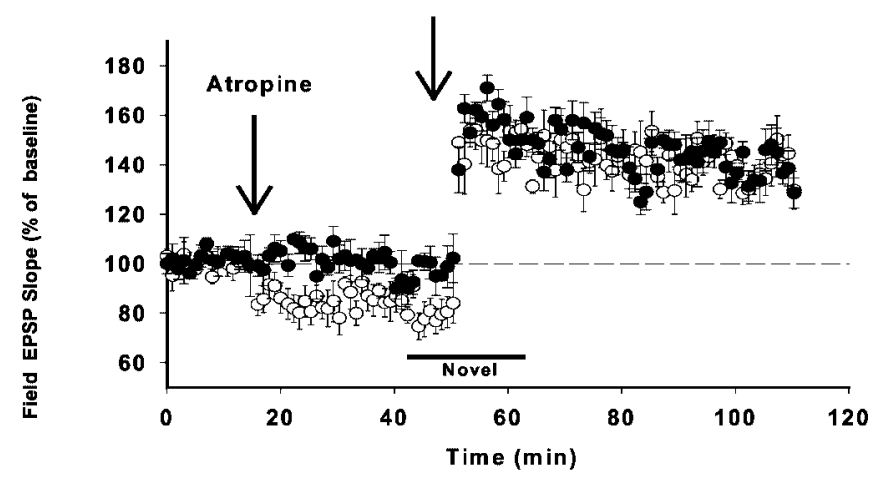

B.

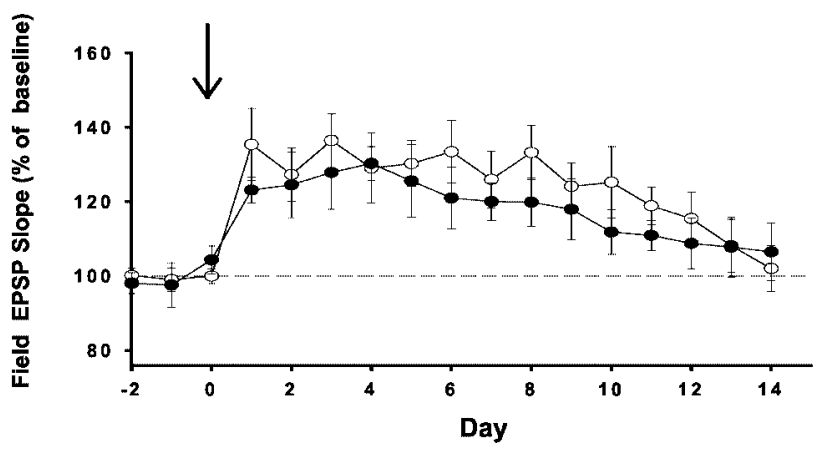

Figure 7. Atropine at a dose that blocks type Il theta rhythm does not alter the facilitation of LTP by novel environments. A, Administration of atropine sulfate $(30 \mathrm{mg} / \mathrm{kg}) 30 \mathrm{~min}$ before inducing LTP in a novel environment attenuated field EPSP but did not alter the facilitation of LTP induction $(O ; n=4)$ compared with control animals $(O ; n=4)$ that received only the water vehicle $(p>0.05)$. $B$, The time course of LTP in animals treated with atropine or vehicle. No significant differences in LTP magnitude at $1 \mathrm{hr}$ or LTP longevity over days 4-14 were observed among atropine- and vehicle-treated animals ( $p>0.05$ ).

normally involved in maintaining memory, these data suggest that conditions associated with learning elicit conditions within the dentate that are optimal for LTP induction.

There is the possibility that the effects of novelty we observed here may have resulted from differences in exploratory activity among animals in novel and familiar environments. However, there were no significant differences in activity among animals exploring home or novel cages, measures of theta rhythm revealed no differences in total theta power during trains, and atropine had no effect on the enhancement of LTP by novelty. Finally, changes in brain temperature were not observed while animals explored either novel or familiar cages. We suspect that the lack of significant changes in activity or brain temperature in animals exploring either novel or familiar environments is likely a result of the novel environments being constructed from standard housing cages that are limited in size. Because a threshold level of activity appears necessary to alter brain temperature and evoked responses (Moser et al., 1994), confining exploration to standard housing cages may have prevented extensive ambulation and increases in brain temperature that can alter evoked responses. Together, these data suggest that novel environments, rather than differences in activity, theta rhythm, or brain temperature, underlie the enhancement of LTP induction and maintenance.

We also noted that the enhancement of LTP when induced in novel cages was significantly attenuated when induced after previous exposure to the novel environment. This suggests that the enhancing effect of novelty on LTP longevity habituates with familiarity to specific environments. This habituation to the novel environment may reflect a general habituation to complex environments or may have resulted from recognition of discrete stimuli within the environment. This was addressed directly in our final experiment. Although the presence of novel objects in a familiar cage had little effect on LTP magnitude, and the probability of LTP induction was reduced after previous exposure to complex environments, the longevity of LTP induced in the presence of novel objects was enhanced. No significant differences in overall activity were observed in these two environments. The reasons for this apparent dissociation of LTP induction and longevity remain to be determined. Although it is possible that different aspects of novel environments differentially affect LTP induction and maintenance, we believe a more likely explanation is that the facilitation of LTP by novelty involves a common process that responds to the overall novelty of the environment. In this view, other stimuli, such as extra cage cues, the orientation of the objects, and so on, might also contribute to the perceived novelty of the environment (Save et al., 1992; Dix and Aggleton, 1999; Wan et al., 1999; O'Reilly and Rudy, 2001; Huxter et al., 2003).

Another effect we observed over the course of these studies was a phasic increase in dentate population spikes accompanied by a paradoxical decreased field EPSP slope. These effects were observed after reorientation in the familiar cage or placement in a novel cage and seem identical to those reported in previous studies (Moser et al., 1994; Moser, 1995, 1996; Paulson and Moser, 1998). This effect is thought to reflect an increase in dendritic inhibition that is accompanied by a concomitant reduction in somatic inhibition in the dentate gyrus and appears to be related to exploratory or investigatory behaviors, rather than specifically to detection of novelty within the environment (Paulsen and Moser, 1998). Nonetheless, such an effect could contribute to new learning, because an overall reduction in synaptic drive, coupled with decreased somatic inhibition, can enhance granule cell output selectively in granule cells receiving the greatest amount of synaptic activation, a process suggested to increase the signal/ noise ratio of granule cell output (Moser, 1996).

Our observation that LTP induced during exposure to novelty enhances the ability to induce a long-lived form of LTP may be relevant to current information-theoretic models of hippocampal information processing. Several of these models suggest that the hippocampus engages in distinct "states" that engage in either the encoding or retrieval of information (Grossberg, 1980; Hasselmo, 1995; Hasselmo and Wyble, 1997; Paulsen and Moser, 1998; Vinogradova, 2001; Hasselmo et al., 2002; Lisman and Otmakhova, 2002). In this view, the hippocampal formation engages in distinct states, with one state operative during learning, whereas the other is thought operative during the recall of previously learned associations (Grossberg, 1980; Treves and Rolls, 1992; Hasselmo and Wyble, 1997; Hasselmo et al., 2002; Lisman and Otmakhova, 2002). This former "learning state" may involve theta states associated with exploration, because theta rhythm has long been implicated in learning, and both theta rhythm and novelty augment information flow through the dentate gyrus (Winson and Abzug, 1978a,b; Harley and Sara, 1992; Kithigina et al., 1997; Yeckel and Berger, 1998; Orr et al., 2001). The activation of the dentate gyrus in response to novelty may be an important feature of learning, because the dentate gyrus is crucial for new learning (McNaughton and Morris, 1987; Barnes, 1988; McNaughton et al., 1989) and is maximally active during the acquisition of novel information (Montag-Sallaz et al., 1999; Zeineh et al., 2003). Given that we observe enhancement of LTP when it is 
induced in novel environments, it appears that novelty elicits conditions at perforant path-dentate synapses that are particularly conducive, and perhaps optimal, for both dentate activity and the induction and maintenance of dentate LTP.

A similar process was posited by Grossberg to solve the "stability-plasticity dilemma" problematic in Hebbian competitive neural networks (Grossberg, 1980; Carpenter and Grossberg, 1988). Stated simply, this dilemma poses the question of how memory systems can encode new information without interfering with previously stored information. This problem can be obviated in real time by normally imposing tight constraints on synaptic plasticity and enhancing synaptic plasticity selectively on detection of novel stimuli (Grossberg, 1980; Murre, 1992). A similar mechanism might be used by the dentate to overcome the stability-plasticity dilemma that is inherent in synaptic systems that use competitive learning (Carpenter and Grossberg, 1988), such as the perforant path-dentate system (Desmond and Levy, 1983; Rolls, 1996; Hasselmo and Wyble, 1997). However, the effects observed here appear too global to allow for synapsespecific enhancement thought necessary for associative learning and are too sustained to permit a rapid assessment of novelty and plasticity as originally envisaged by Grossberg (1980). Perhaps the combination of a sustained and global facilitation of LTP by novelty, combined with the enhanced discrimination of dentate output observed during the first few minutes of exploration (Fig. 2) (Moser, 1996), act in concert to allow synapse-specific LTP facilitation. In this view, the global facilitation effect of novelty would enhance synaptic plasticity only in those granule cells receiving the greatest synaptic input. The combination of these two phenomena could provide a potential mechanism for a synapsespecific facilitation of LTP by novelty, a mechanism that also could preserve the stability of previously stored patterns by selectively enhancing plasticity only at synapses on active granule cells.

Different afferent systems of the hippocampus can display distinct mechanisms of LTP induction (Harris and Cotman, 1986; Derrick and Martinez, 1989; Bramham et al., 1991; Grover and Teyler, 1995; Do et al., 2002). The present data extend these findings to indicate that LTP displaying distinct properties, such as enhanced longevity, can be induced in distinct behavioral states even within a single synaptic population. Thus, our data suggest that LTP at a given synapse can display different time courses, and possibly different "forms," of L-LTP maintenance. Importantly, the expression of this long-lived form of LTP appears to be determined by the behavioral state of the animal during LTP induction.

At the perforant path-dentate synapse, LTP can last from weeks to months, a finding that has been used as an argument both for (Barnes, 1988; Martinez and Derrick, 1996) and against (Shors and Matzel, 1997) LTP as a mechanism of sustained information storage. However, as is suspected with the human hippocampus, the contribution of the hippocampal formation in memory storage in the rat also is thought to be time-limited, because post-training hippocampal lesions appear to have little effect on spatial memory when made several weeks after training (Barnes, 1988). Our data indicate a robust increase in the longevity of LTP to several weeks when it is induced during conditions that are associated with learning. Thus, the duration of LTP induced in this state approximately parallels the period of time in which the hippocampal formation is necessary for memory, a finding that provides additional support to the view that LTP within afferents of the hippocampal formation is of sufficient duration, and thus is a viable candidate mechanism, for sustaining recently acquired hippocampus-based memory.

\section{References}

Abraham WC, Logan B, Greenwood JM, Dragunow M (2002) Induction and experience-dependent consolidation of stable long-term potentiation lasting months in the hippocampus. J Neurosci 22:9626-9634.

Arai A, Lynch G (1992) Factors regulating the magnitude of long-term potentiation induced by theta pattern stimulation. Brain Res 598:173-184.

Barnes CA (1979) Memory deficits associated with senescence: a neurophysiological and behavioral study in the rat. J Comp Physiol Psychol 93:74-104.

Barnes CA (1988) Spatial learning and memory processes: the search for their neurobiological mechanisms in the rat. Trends Neurosci 11:163-169.

Bliss TVP, Collingridge GL (1993) A synaptic model of memory: long-term potentiation in the hippocampus. Nature 361:31-39.

Bliss TVP, Gardner-Medwin AR (1973) Long-lasting potentiation of synaptic transmission in the dentate area of the unanaesthetized rabbit following stimulation of the perforant path. J Physiol (Lond) 232:357-374.

Bliss TVP, Lomo T (1973) Long-lasting potentiation of synaptic transmission in the dentate area of the anesthetized rabbit following stimulation of the perforant path. J Physiol (Lond) 232:331-356.

Bramham CR, Srebro B (1989) Synaptic plasticity in the hippocampus is modulated by behavioral state. Brain Res 493:74-86.

Bramham CR, Milgram NW, Srebro B (1991) Activation of AP5-sensitive NMDA receptors is not required to induce LTP of synaptic transmission in the lateral perforant path. Eur J Neurosci 3:1300-1308.

Buzsaki G (2002) Theta oscillations in the hippocampus. Neuron 33:325-340.

Buzsaki G, Grastyan E, Czopf J, Kellenyi L, Prohaska O (1981) Changes in neuronal transmission in the rat hippocampus during behavior. Brain Res 225:235-247.

Carpenter GA, Grossberg S (1988) The art of adaptive pattern recognition by a self-organising neural network. IEEE Comput 21:77-88.

Chaillan FA, Roman FS, Soumireu-Mourat B (1996) Modulation of synaptic plasticity in the hippocampus and piriform cortex by physiologically meaningful olfactory cues in an olfactory association task. J Physiol (Paris) 90:343-347.

Davis-Hart C, Derrick BE (1997) Exposure to a novel environment facilitates LTP induction at medial perforant path-dentate synapses in awake, freely moving animals. Soc Neurosci Abstr 23:788.

Davis-Hart C, Derrick BE (1999) Novelty-induced facilitation of LTP: independence from exploratory behavior and dependence on novel stimuli. Soc Neurosci Abstr 25:897.

Davis-Hart C, Bailey K, Heath T, Derrick BE (1998) Novelty-induced facilitation of LTP: independence form a preceding attenuation effect and brain temperature. Soc Neurosci Abstr 24:332.

Davis-Hart CD, Derrick BE (2000) Novelty-induced facilitation of LTP is not modulated by acetylcholine or norepinephrine. Soc Neurosci Abstr 26:751.1.

Derrick BE, Martinez Jr JL (1989) A unique, opioid peptide-dependent form of long-term potentiation is found in the CA3 region of the rat hippocampus. Adv Biosci 75:213-216.

Desmond NL, Levy WB (1983) Synaptic correlates of associative potentiation/ depression: an ultrastructural study in the hippocampus. Brain Res 265:21-30.

Diamond DM, Rose GM (1994) Stress impairs LTP and hippocampaldependent memory. Ann NY Acad Sci 746:411-414.

Dix SL, Aggleton JP (1999) Extending the spontaneous preference test of recognition: evidence of object-location and object-context recognition. Behav Brain Res 99:191-200.

Do V, Martinez CO, Martinez Jr JLM, Derrick BE (2002) Long-term potentiation in direct perforant path projections to hippocampal area CA3 in vivo. J Neurophysiol 87:669-674.

Erickson CA, McNaughton BL, Barnes CA (1993) Comparison of longterm enhancement and short-term exploratory modulation of perforant path synaptic transmission. Brain Res 615:275-280.

Green EJ, Greenough WT (1986) Altered synaptic transmission in dentate gyrus of rats reared in complex environments: evidence from hippocampal slices maintained in vitro. J Neurophysiol 55:739-750.

Grossberg S (1980) How does the brain build a cognitive code? Psychol Rev 87:1-51.

Grover LM, Teyler TJ (1995) Different mechanisms may be required for maintenance of NMDA receptor-dependent and independent forms of long-term potentiation. Synapse 19:121-133.

Harley CW, Sara SJ (1992) Locus coeruleus bursts induced by glutamate trigger delayed perforant path strike amplitude potentiation in the dentate gyrus. Exp Brain Res 89:581-587. 
Harris EW, Cotman CW (1986) Long-term potentiation of guinea pig mossy fiber responses is not blocked by $N$-methyl D-aspartate antagonists. Neurosci Lett 70:132-137.

Hasselmo ME (1995) Dynamics of learning and recall at excitatory recurrent synapses and cholinergic modulation in rat hippocampal region CA3. J Neurosci 15:5249-5262.

Hasselmo ME, Wyble BP (1997) Free recall and recognition in a network model of the hippocampus: simulating effects of scopolamine on human memory function. Behav Brain Res 89:1-34.

Hasselmo ME, Bodelon C, Wyble BP (2002) A proposed function for hippocampal theta rhythm: separate phases of encoding and retrieval enhance reversal of prior learning. Neural Comput 14:793-817.

Huerta PT, Lisman JE (1993) Heightened synaptic plasticity of hippocampal CA1 neurons during a cholinergically induced rhythmic state. Nature 364:723-725.

Huxter J, Burgess N, O’Keefe J (2003) Independent rate and temporal coding in hippocampal pyramidal cells. Nature 425:828-832.

Jones-Leonard B, McNaughton BL, Barnes CA (1987) Suppression of hippocampal synaptic plasticity during slow-wave sleep. Brain Res 425:174-177.

Kithigina V, Vankov A, Harley C, Sara SJ (1997) Novelty-elicited, noradrenaline-dependent enhancement of excitability in the dentate gyrus. Eur J Neurosci 9:41-47.

Klukowski G, Harley CW (1994) Locus coeruleus activation induces perforant path-evoked population spike potentiation in the dentate gyrus of awake rat. Exp Brain Res 102:165-170.

Landfield PW, McGaugh JL, Tusa RJ (1972) Theta rhythm: a temporal correlate of memory storage processes in the rat. Science 175:87-89.

Li S, Cullen WK, Anwyl R, Rowan MJ (2003) Dopamine-dependent facilitation of LTP induction in hippocampal CA1 by exposure to spatial novelty. Nat Neurosci 6:526-531.

Lisman JE, Otmakhova NA (2001) (2002) Storage, recall, and novelty detection of sequences by the hippocampus: elaborating on the SOCRATIC model to account for normal and aberrant effects of dopamine. Hippocampus 11:551-568.

Martinez JL, Derrick BE (1996) Long-term potentiation and learning. Annu Rev Psychol 47:173-203.

McNaughton BL (1980) Evidence for two physiologically distinct perforant pathways to the fascia dentate. Brain Res 199:1-19.

McNaughton BL, Barnes CA (1977) Physiological identification and analysis of dentate granule cell responses to stimulation of the medial and lateral perforant pathways in the rat. J Comp Neurol 175:439-454.

McNaughton BL, Morris RGM (1987) Hippocampal synaptic enhancement and information storage within a distributed memory system. Trends Neurosci 10:408-415.

McNaughton BL, Barnes CA, Meltzer J, Sutherland RJ (1989) Hippocampal granule cells are necessary for normal spatial learning but not for spatially-selective pyramidal cell discharge. Exp Brain Res 76:485-496.

Montag-Sallaz M, Welzl H, Kuhl D, Montag D, Schachner M (1999) Novelty-induced increased expression of immediate-early genes c-fos and arg 3.1 in the mouse brain. J Neurobiol 38:234-246.

Moser E, Mathiesen I, Andersen P (1993) Association between brain temperature and dentate field potentials in exploring and swimming rats. Science 259:1324-1326.

Moser EI (1995) Learning-related changes in hippocampal field potentials. Behav Brain Res 71:11-18.

Moser EI (1996) Altered inhibition of dentate granule cells during spatial learning in an exploration task. J Neurosci 16:1247-1259.

Moser EI, Moser MB, Andersen P (1994) Potentiation of dentate synapses initiated by exploratory learning in rats: dissociation from brain temperature, motor activity, and arousal. Learn Mem 1:55-73.

Murre JMJ (1992) Learning and categorization in modular neural networks. New York: Erlbaum.

O'Keefe J, Nadel L (1978) The hippocampus as a cognitive map. London: Oxford UP.

O’Reilly RC, Rudy JW (2001) Conjunctive representations in learning and memory: principles of cortical and hippocampal function. Psychol Rev 108:311-345.

Orr G, Rao G, Houston FP, McNaughton BL, Barnes CA (2001) Hippocampal synaptic plasticity is modulated by theta rhythm in the fascia dentata of adult and aged freely behaving rats. Hippocampus 11:647-654.

Pavlides C, Greenstein YJ, Grudman M, Winson J (1988) Long-term poten- tiation in the dentate gyrus is induced preferentially on the positive phase of theta-rhythm. Brain Res 439:383-387.

Paxinos G, Watson C (1982) Sterotaxic atlas of the rat brain. New York: Academic.

Rolls ET (1996) A theory of hippocampal function in memory. Hippocampus 6:601-620.

Roman F, Staubli U, Lynch G (1987) Evidence for synaptic potentiation in a cortical network during learning. Brain Res 418:221-226.

Rosenzweig M, Krech D, Bennet EL, Diamond M (1962) Effects of environmental complexity and training on brain chemistry and anatomy: a replication and extension. J Comp Physiol Psychol 55:429-437.

Sara SJ, Segal M (1991) Plasticity of sensory responses of locus coeruleus neurons in behaving rats: implication for cognition. Prog Brain Res 88:571-585.

Save E, Poucet B, Foreman N, Buhot MC (1992) Object exploration and reactions to spatial and nonspatial changes in hooded rats following damage to parietal cortex or hippocampal formation. Behav Neurosci 106:447-456.

Sharp PE, McNaughton BL, Barnes CA (1985) Enhancement of hippocampal field potentials in rats exposed to a novel, complex environment. Brain Res 339:361-365.

Shors TJ, Matzel LD (1997) Long-term potentiation: what's learning got to do with it? Behav Brain Sci 20:597-614.

Stanton PK, Sarvey JM (1987) Norepinephrine regulates long-term potentiation of both the population spike and dendritic EPSP in hippocampal dentate gyrus. Brain Res Bull 18:115-119.

Staubli U, Lynch G (1987) Stable long-term potentiation elicited by theta pattern stimulation. Brain Res 435:227-234.

Straube T, Korz V, Frey J (2003a) Bidirectional modulation of long-term potentiation by novelty-exploration in rat dentate gyrus. Neurosci Lett 344:5-8.

Straube T, Korz V, Balschun D, Frey JU (2003b) Requirement of $\beta$-adrenergic receptor activation and protein synthesis for LTP-reinforcement by novelty in rat dentate gyrus. J Physiol (Lond) 552:953-960.

Treves A, Rolls ET (1992) Computational constraints suggest the need for two distinct input systems to the hippocampal CA3 network. Hippocampus 2:189-199.

Vanderwolf CH (1975) Neocortical and hippocampal activation in relation to behavior: effects of atropine, eserine, phenothiazines, and amphetamine. J Comp Physiol Psychol 88:306-323.

Vanderwolf CH, Leung LS (1983) Hippocampal rhythmical slow activity: a brief history and the effects of entorhinal lesions and phencyclidine. In: Neurobiology of the hippocampus (Seifert GW, ed), pp 275-302. New York: Academic.

Vankov A, Herve-Minivielle A, Sara SJ (1995) Response to novelty and its rapid habituation in locus coeruleus neurons of the freely exploring rat. Eur J Neurosci 7:1180-1187.

Villarreal DM, Do V, Haddad E, Derrick BE (2002) NMDA antagonists sustain LTP and spatial memory: active processes mediate LTP decay. Nat Neurosci 5:48-52.

Vinogradova OS (2001) Hippocampus as comparator: role of the two input and two output systems of the hippocampus in selection and registration of information. Hippocampus 11:578-598.

Wan H, Aggleton JP, Brown MW (1999) Different contributions of the hippocampus and perirhinal cortex to recognition memory. J Neurosci 19:1142-1148.

Wigstrom H, Gustafsson B (1983) Large long-lasting potentiation in the dentate gyrus in vitro during blockade of inhibition. Brain Res 275:153-158.

Wigstrom H, Gustaffson B (1985) Facilitation of hippocampal long-lasting potentiation by GABA antagonists. Acta Physiol Scand 125:159-172.

Wilson MA, McNaughton BL (1993) Dynamics of the hippocampal ensemble code for space. Science 261:1055-1058.

Winson J, Abzug C (1978a) Dependence upon behavior of neuronal transmission from perforant pathway through entorhinal cortex. Brain Res 147:422-427.

Winson J, Abzug C (1978b) Neuronal transmission through hippocampal pathways dependent upon behavior. J Neurophys 41:716-732.

Yeckel MF, Berger TW (1998) Spatial distribution of potentiated synapses in hippocampus: dependence on cellular mechanisms and network properties. J Neurosci 18:438-450.

Zeineh MM, Engel SA, Thompson PM, Bookheimer SY (2003) Dynamics of the hippocampus during encoding and retrieval of face-name pairs. Science 299:577-580. 